\title{
EFEITOS DO MAT PILATES EM PACIENTES COM DOR LOMBAR DEVIDO A DISCOPATIA DEGENERATIVA: RELATOS DE CASOS
}

\section{Effects of Mat Pilates in patients with low back pain from degenerative discopaty: a case study}

Natália M. Castanhetti ${ }^{1}$, Liliana Sudré-Marques ${ }^{1}$, Lee Gi Fan ${ }^{1}$

1Universidade do Extremo Sul Catarinense, Unidade Acadêmica da Saúde, Curso de Fisioterapia.

Projeto aprovado pelo Comitê de Ética em Pesquisa da Universidade do Extremo Sul Catarinense, protocolo número: 1.283 .220

\section{Endereço para Correspondência:}

Lee Gi Fan

Universidade do Extremo Sul Catarinense, Unidade Acadêmica da Saúde, Curso de Fisioterapia. Avenida Universitária, 1105 - Bairro Universitário. CEP: 88806-000 Criciúma/SC.

Fone/ Fax: +55(48) 34312500 .

Email: Igf@unesc.net 


\title{
Resumo
}

A discopatia degenerativa lombar vem se tornando um problema mundial por causa da incapacidade, disfunções e problemas socioeconômicos e necessita ser melhor estudada visando prevenção e tratamento. $O$ objetivo do estudo foi analisar o efeito do Método Pilates em pacientes que sofrem de discopatia degenerativa lombar. $\mathrm{O}$ Método Pilates foi realizado no solo, como terapia, por um tempo determinado e com um protocolo estabelecido. O estudo foi realizado com três pacientes com discopatia degenerativa lombar que foram avaliados antes e após a intervenção da terapia com o Método por um período de dois meses com frequência de duas vezes por semana, totalizando 15 sessões. Foi utilizado um protocolo elaborado com oito exercícios realizados em solo. Os testes realizados foram: Laségue e Valsalva para verificar dor irradiada; Questionário Nórdico de Sintomas Osteomusculares (QNSO) e a Escala Visual Analógica (EVA) para avaliar e quantificar a dor dos pacientes referente à região lombar; e teste de força abdominal. Os resultados deste estudo evidenciaram que a aplicação do Método Pilates foi importante na redução da dor lombar, aumento da força muscular abdominal dos pacientes e foram obtidas modificações positivas nos resultados do EVA e QNSO. O Método Pilates se mostrou eficaz na melhora da dor lombar e força muscular abdominal.

Palavras-chave: Discopatia Degenerativa; Protrusão Discal; Hérnia de Disco; Método Pilates; Dor Lombar.

\begin{abstract}
Lumbar Degenerative disc disease is becoming a global problem because of the disability, dysfunction and Socioeconomic problems and needs further studied aiming prevention and treatment. The study goal was to analyze the Pilates Method effect in patients suffering of lumbar degenerative disc disease; the Pilates Method was apply in the soil, as therapy for a determined time interval and established protocol. The study was conducted with three patients with lumbar degenerative disc disease, evaluated before and after therapy Intervention with method for a period of two months with frequency of twice a week, totaling 15 sessions. We used a prepared protocol with eight exercises done in soil. The clinical tests used were: Lasègue and Valsalva to verify radiated pain; Nordic Musculoskeletal Questionnaire (NMQ) and a Visual
\end{abstract}




\section{Artigo Original}

\section{Saúde Funcional}

Analogue Scale (VAS) to evaluate and quantify the pain of patients in lumbar region; and abdominal strength test. The results of this study indicated that the Pilates Method application was important in the reduction of low back pain, increased abdominal muscle strength of patients and positive changes were obtained on VAS and NMQ results. The Pilates Method has showed effective in improving low back pain and abdominal muscle strength.

Keywords: Degenerative Disk Disease; Disc Protrusion; Disc Herniation; Pilates Method; Low Back Pain.

\section{INTRODUÇÃO}

Discopatia degenerativa é caracterizada como a expressão do estado do disco intervertebral, sendo o resultado de etiologias variadas que atuam individualmente ou associadas entre $\mathrm{si}^{1}$. Alterações degenerativas podem causar danos a estas estruturas, tais como: estenose; doença degenerativa do disco; hérnia de disco; bem como qualquer deficiência degenerativa do arco posterior, por desencadear a compressão de estruturas neurais e a anormalidades dos vasos ${ }^{2,3}$.

A doença degenerativa do disco tem etiologias variadas, podendo ter sua origem em condições diferentes, como hérnia discal e espondilose. Os sintomas manifestam-se sob a forma de três síndromes álgicas: dor axial, dor radicular, mielopatia ou ainda com associação destas ${ }^{4-7}$.

Os desequilíbrios musculares, traumas, esforços nas atividades diárias e posturas que facilitam a desorganização da distribuição das pressões do disco, são alguns dos mecanismos que favorecem a degeneração. Dessa forma, a má postura, musculatura fraca, posturas em pé por períodos prolongados, levantamento excessivo de peso e trabalhos repetitivos podem lesionar a coluna lombar, desencadeando a discopatia degenerativa lombar ${ }^{8,9}$.

A Hérnia de disco é definida como um deslocamento localizado do núcleo pulposo para além dos limites do espaço do disco intervertebral, podendo assumir a forma de protrusão ou extrusão ${ }^{10}$. A protrusão ou abaulamento discal é o início de um processo de deslocamento do disco sem extravasamento do material interno, enquanto que a extrusão e sequestro é um estágio final deste processo. Protrusão 


\section{Artigo Original \\ Saúde Funcional}

discal sem fragmento herniado ou defeito no anel fibroso é descrito como estágio que antecede a hérnia de disco no processo de degeneração do disco intervertebral ${ }^{11,12}$.

O método Pilates é definido como uma técnica que se concentra na postura, concentração, respiração, flexibilidade, força e controle muscular ${ }^{13}$. A abordagem do método Pilates concentra-se no fortalecimento de músculos do tronco para estabilização da região pélvico-lombar ${ }^{14}$. Estes exercícios têm por objetivo melhorar a estabilidade estática e dinâmica, bem como a postura e movimentos em geral ${ }^{15}$. O método consiste em contrações musculares isométricas conhecidos como Power house, responsável pela estabilização da coluna vertebral e da pelve na postura estática, auxiliando na estabilidade dinâmica do corpo durante os exercícios ${ }^{16,17}$.

Sabemos que os grupos musculares exercem papéis fundamentais em cada parte do corpo na qual se localizam e, assim como todos os grupos musculares, os músculos abdominais têm um papel muito importante, eles participam no suporte da coluna vertebral fazendo uma diminuição na tensão que é exercida sobre a ela. Sendo assim, com o enfraquecimento de tal musculatura esse suporte poderá ser diminuído se fortalecido ${ }^{18}$. Os exercícios capazes de fortalecer os músculos abdominais aumentam a estabilidade da coluna vertebral. Existem evidências convincentes de que a realização de um Método com foco no fortalecimento da musculatura extensora do tronco restaura a função da coluna lombar e pode prevenir o surgimento de dores ${ }^{19}$. Uma das técnicas que está sendo usada pelos fisioterapeutas é o Método Pilates ${ }^{20}$. Baseado nisso, o presente estudo tem como objetivo analisar os efeitos do Método Mat Pilates sobre a dor em indivíduos que apresentam discopatias degenerativas da coluna lombar.

\section{MATERIAIS E MÉTODOS}

\section{Aspectos Éticos}

A presente pesquisa foi aprovada pelo Comitê de Ética da Universidade do Extremo Sul Catarinense. No primeiro contato foi explicado para os pacientes os objetivos da pesquisa e, posteriormente, foram convidados a participar do estudo. Aqueles que aceitaram fazer parte da pesquisa concordaram e assinaram o termo de 
Artigo Original

Saúde Funcional

consentimento livre e esclarecido (TCLE). No caso de dúvidas em relação ao conteúdo do TCLE, as mesmas foram esclarecidas pelas pesquisadoras.

\section{Amostra}

A amostra desta pesquisa caracteriza-se como sendo não probabilística, por conveniência. Os integrantes da pesquisa foram pacientes com diagnóstico de discopatia degenerativa lombar, de ambos os sexos, entre 25 a 35 anos, com queixa de dor lombar. Essa amostra foi composta por 3 pacientes no total, caracterizando 0 estudo como estudo de caso.

\section{Critérios para Inclusão}

Foram incluídos nesta pesquisa os pacientes previamente selecionados que possuíam diagnóstico de discopatia degenerativa com seguintes características:

1) Que apresentavam dor lombar;

2) Que não estavam em quadro álgico agudo;

3) Que não estavam fazendo nenhum tipo de tratamento físico;

4) Que não estavam fazendo tratamento medicamentoso;

5) Que apresentaram atestado médico para realização do Método Pilates;

6) Indivíduos de ambos os sexos;

7) Com idade entre 25 a 35 anos;

8) Que concordaram e assinaram o TCLE.

\section{Critérios para Exclusão}

Foram excluídos da pesquisa os indivíduos que não concordassem em assinar o TCLE, indivíduos que praticassem atividades físicas, como musculação, yoga, caminhada e outras, ou que não se encaixassem aos critérios de inclusão. 
Artigo Original

Saúde Funcional

\section{Local de Execução}

Os procedimentos foram realizados no laboratório de habilidades do Serviço de Fisioterapia nas Clínicas Integradas da UNESC.

\section{Delineamento Experimental}

Depois de selecionados os participantes da pesquisa, os mesmos receberam todas as informações de como seria realizado o Método Pilates.

\section{Método Pilates}

O Método Pilates foi realizado no solo (Método Mat Pilates). Para a execução dos procedimentos, foram necessários os seguintes materiais: bola suíça e colchonete. Os exercícios do Método Pilates que foram selecionados para serem realizados neste estudo são: The Hundred, Double LegsTretch, Criss Cross, OneLegCircle, LegLifts, The Side Bend, SquatWith Ball, The Spine Twist, conforme apresentado no protocolo de aplicação.

Os exercícios propostos tiveram como objetivos: fortalecer a musculatura reto abdominal, transverso abdominal, oblíquos, reto femoral, glúteo máximo e médio, adutores, quadrado lombar; mobilizar a coluna lombar; alongar os músculos paravertebrais, rotadores do tronco, isquiotibiais e quadrado lombar e melhorar a mobilidade e a estabilidade rotatória do quadril.

A técnica foi realizada 2 vezes por semana, totalizando 15 sessões, com o tempo estimado de 40 minutos. Os pacientes foram atendidos individualmente com horários previamente estabelecidos.

\section{Teste de Valsalva}

Para avaliar a dor na coluna lombar foi realizado o teste de Valsalva. Este teste foi realizado com o paciente sentado e foi pedido para que ele prendesse a respiração e fizesse força como se fosse evacuar. Esta ação leva a um aumento da pressão intratecal, ocasionando dor em pacientes com alterações na coluna lombar. 
Artigo Original

Saúde Funcional

Após o paciente realizar a ação, perguntava-se se havia agravamento da dor com esta. Foi considerado positivo se o paciente apresentasse dor ${ }^{21}$.

\section{Teste de Laségue}

Para avaliar a irradiação da dor para membros inferiores foi realizado o teste de Laségue. Durante este teste, o paciente é posicionado em decúbito dorsal e relaxado. $O$ teste consiste na elevação do membro inferior do paciente com o joelho estendido, enquanto que o pesquisador segura o tornozelo. $O$ teste é considerado positivo quando reproduz dor entre $30^{\circ}$ e $60^{\circ}$ de elevação tanto na região lombar quanto no membro inferior ${ }^{22}$.

\section{Questionário Nórdico de Sintomas Osteomusculares}

O Questionário Nórdico de Sintomas Osteomusculares (QNSO) foi desenvolvido com a proposta de padronizar a mensuração de relato de sintomas osteomusculares e, assim, facilitar a comparação dos resultados entre os estudos. Este questionário não é indicado como base para diagnóstico clínico, mas para a identificação de distúrbios osteomusculares e, como tal, pode constituir importante instrumento de diagnóstico do ambiente ou do posto de trabalho. Há três formas do QNSO: uma forma geral, compreendendo todas as áreas anatômicas, e outras duas específicas para as regiões lombar e de pescoço e ombros. O instrumento consiste em escolhas múltiplas ou binárias quanto à ocorrência de sintomas nas diversas regiões anatômicas nas quais são mais comuns. $O$ respondente deve relatar a ocorrência dos sintomas considerando os 12 meses e os sete dias precedentes à entrevista, bem como relatar a ocorrência de afastamento das atividades rotineiras no último ano. No presente estudo o QNSO foi adaptado, a partir do instrumento trazido por Barros e Alexandre ${ }^{23}$, para avaliação apenas da coluna lombar e membros inferiores ${ }^{23}$. 
Artigo Original

Saúde Funcional

\section{Escala Visual Analógica (EVA)}

No início de cada sessão do Método Pilates a dor lombar foi avaliada por meio da Escala visual analógica (EVA). Trata-se de uma linha com as extremidades numeradas de 0-10. Em uma extremidade da linha é marcada "nenhuma dor" e na outra "pior dor imaginável". Pede-se, então, para que o paciente avalie e marque na linha a dor presente naquele momento ${ }^{24}$.

\section{Teste de Força Abdominal}

No teste abdominal coloca-se o indivíduo em decúbito dorsal, com o joelho flexionado a $90^{\circ}$ e com os braços cruzados sobre o tórax, executando uma flexão do tronco, encostando os cotovelos na coxa e retornando à posição inicial. O paciente teve 1 minuto para realizar o maior número de repetições. A quantidade de repetições feitas pelo indivíduo foi anotada e comparada de acordo com a tabela padrão de força abdominal, apresentada abaixo ${ }^{25}$.

TESTE DE FORÇA ABDOMINAL (repetições em 1 minuto)

\begin{tabular}{|lccccc|}
\hline \multicolumn{5}{c|}{ HOMENS } \\
\hline Idade & excelente & Bom & médio & regular & Fraco \\
\hline $20-29$ & $>48$ & $43-47$ & $37-42$ & $33-36$ & $0-32$ \\
$30-39$ & $>40$ & $35-39$ & $29-34$ & $25-38$ & $0-24$ \\
$40-49$ & $>35$ & $30-34$ & $24-29$ & $20-23$ & $0-19$ \\
$50-59$ & $>30$ & $25-29$ & $19-24$ & $15-18$ & $0-14$ \\
$60-69$ & $>25$ & $20-24$ & $14-19$ & $10-13$ & $0-9$ \\
\hline & $>44$ & $39-43$ & $33-38$ & $29-32$ & $0-38$ \\
\hline $20-29$ & $>36$ & $31-35$ & $25-30$ & $21-24$ & $0-20$ \\
$30-39$ & $>31$ & $26-30$ & $19-25$ & $16-18$ & $0-15$ \\
$40-49$ & $>26$ & $21-25$ & $15-20$ & $11-14$ & $0-10$ \\
$50-59$ & $>21$ & $16-20$ & $10-15$ & $06-09$ & $0-5$ \\
$60-69$ & & & & \\
\hline
\end{tabular}

\section{Análise de Dados}

Os dados coletados foram organizados em planilhas do software Microsoft Excel versão 2010. A evolução dos escores da escala (EVA) foi avaliada por meio da 
Artigo Original

Saúde Funcional

construção de um gráfico de linhas. Foi calculada média e desvio padrão para a referida escala, cujos resultados foram expressos por meio de gráfico de média e barra de erro. Os resultados do teste de força abdominal foram expressos por meio de gráfico de colunas.

\section{RESULTADOS}

\section{Resultados dos Dados gerais}

A amostra foi composta por três pacientes com queixa de dor lombar, sendo dois do sexo feminino (relatados aqui como paciente 1 e paciente 2 ) e um do sexo masculino (relatado aqui como paciente 3). A idade do paciente 1 foi de 35 anos, do paciente 2 de 30 anos e do paciente 3 de 25 anos.

\section{Resultados do teste de Valsalva e Laségue}

A manobra de Valsalva e o teste de Laségue apresentaram resultado positivo antes e após o Método Pilates, em todos os pacientes avaliados, demonstrando que a irradiação da dor lombar e dos membros inferiores permaneciam, mesmo após o método.

\section{Questionário Nórdico de Sintomas Osteomusculares}

Os resultados do questionário Nórdico estão representados na Tabela 1. A primeira questão levantada aos pacientes foi sobre aspectos relacionados a problemas como dor, desconforto ou dormência nos últimos doze meses antes da iniciação do Método Pilates. O paciente 1 e o paciente 3 relataram ter algum tipo de problema na região lombar com frequência e o paciente 2 relatou sentir dor raramente. Quanto aos membros inferiores, o paciente 1 afirmou sentir dor com frequência, o paciente 2 raramente e o paciente 3 sempre.

A segunda questão está relacionada a problemas como dor, desconforto ou dormência nos últimos sete dias antes da iniciação do Método Pilates. O paciente 1 relatou raramente ter algum tipo de problema na região lombar, o paciente 2 relatou 


\section{Artigo Original}

\section{Saúde Funcional}

sempre e o paciente $3 \mathrm{com}$ frequência. Quanto a problemas relacionados aos membros inferiores, o paciente 1 afirmou que isso ocorre raramente, o paciente 2 relatou não ter tido nenhum problema e o paciente 3 sempre.

A terceira questão está relacionada aos aspectos de impedimento de realização das atividades nos últimos 12 meses antes da iniciação do Método Pilates. O paciente 1 relatou não ter problemas relacionados à região lombar. Entretanto, os pacientes 2 e 3 relataram problemas relacionados à região lombar. Os pacientes $1 \mathrm{e}$ 2 afirmaram não ter tido problemas relacionados aos membros inferiores. Por outro lado, o paciente 3 relatou que teve problemas relacionados aos membros inferiores.

A última pergunta da tabela foi aplicada após o Método Pilates. A pergunta está relacionada aos problemas como dor, desconforto ou dormência nos últimos sete dias. Todos os pacientes relataram ter raramente algum tipo de problema na região lombar. Quanto a problemas relacionados aos membros inferiores, os pacientes 1 e 3 relataram que isso ocorre raramente, o paciente 2 relatou não ter problema.

\section{Escala Visual Analógica - EVA}

Os pacientes foram avaliados através da Escala Visual Analógica (EVA) (Figura 1) e também através da avaliação da dor antes da primeira sessão do Método Pilates e após a última sessão (Figura 2), sendo que o paciente 1 relatou dor grau quatro na primeira sessão e dor grau zero na última, o paciente 2 relatou dor grau cinco na primeira sessão e dor grau um na última, e o paciente 3 relatou dor grau sete na primeira sessão e dor grau três na última.

\section{Teste de força Abdominal}

A figura 3 representa os resultados de força abdominal. Como pode ser observado, o paciente 1 apresentava força abdominal abaixo da média na primeira avaliação. Após o Método Pilates este paciente teve um considerável ganho de força, apresentando na última avaliação força abdominal acima da média. O paciente 2 já apresentava força abdominal acima da média antes do Método Pilates. Após a aplicação deste método, a paciente obteve ganho de força significativa, apresentando resultado excelente, de acordo com a tabela de referência. Já o paciente 3 , após a 
Artigo Original

Saúde Funcional

aplicação do Método Pilates, passou de fraco para abaixo da média sua força abdominal, apresentando também ganho de força após o método.

Tabela 1. Resultados descritivos referente ao questionário Nórdico antes e depois do Pilates. Sendo que 0 é igual a não, 1 é igual a Raramente, 2 é igual a Com frequência, 3 é igual a Sempre.

\section{QUESTIONÁRIO NÓRDICO ANTES DO PILATES}

Considerando os últimos 12 meses, você tem tido algum problema (tal como Dor, desconforto ou Paciente 1 Paciente 2 Paciente 3 dormência)?

Lombar

Quadril e Membros inferiores
Frequência Raramente Frequência

Frequência Raramente Sempre

Considerando os últimos 7 dias, você tem tido algum problema (tal como Dor, desconforto ou Paciente 1 Paciente 2 Paciente 3 dormência)?

Lombar

Raramente Sempre Frequência

Quadril e Membros inferiores Raramente Não Sempre

Durante os últimos 12 meses se o paciente teve algum impedimento para realizar suas atividades (trabalho, esportes, trabalho em casa...) por

Paciente 1 Paciente 2 Paciente 3 causa do seu problema.

Relacionado a dor lombar

Não

Sim

Sim

Relacionado a quadril e Membros inferiores

Não

Não

Sim

\section{QUESTIONÁRIO NÓRDICO DEPOIS DO PILATES}

Considerando os últimos 7 dias, você tem tido algum problema (tal como Dor, desconforto ou Paciente 1 Paciente 2 Paciente 3 dormência)?

Lombar

Quadril e Membros inferiores
Raramente Raramente Raramente

Raramente Não Raramente 


\section{Artigo Original}

\section{Saúde Funcional}

Figura 1. Análise da dor ao longo das sessões conforme EVA, utilizando uma escala de 0 a 10, sendo considerado 0 = ausência de dor e $10=$ dor máxima. Cada sessão teve duração $=40$ minutos, realizadas 2 vezes por semana, somando um total de 15 sessões.

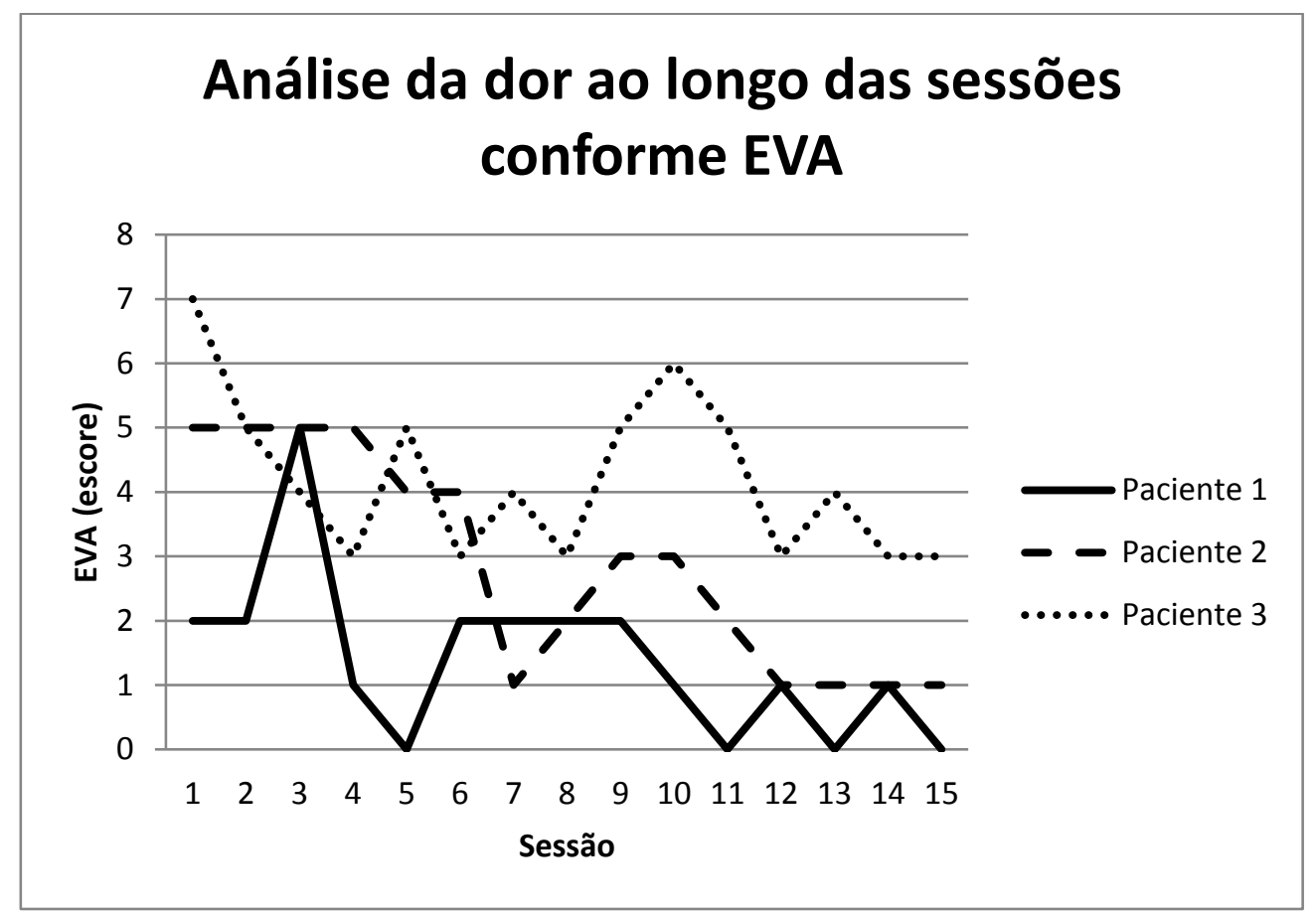

Figura 2. Avaliação da dor antes da primeira sessão do Método Mat Pilates e depois da última sessão. Foi utilizada uma escala de 0 a 10, sendo considerado $0=$ ausência de dor e 10 = dor máxima.

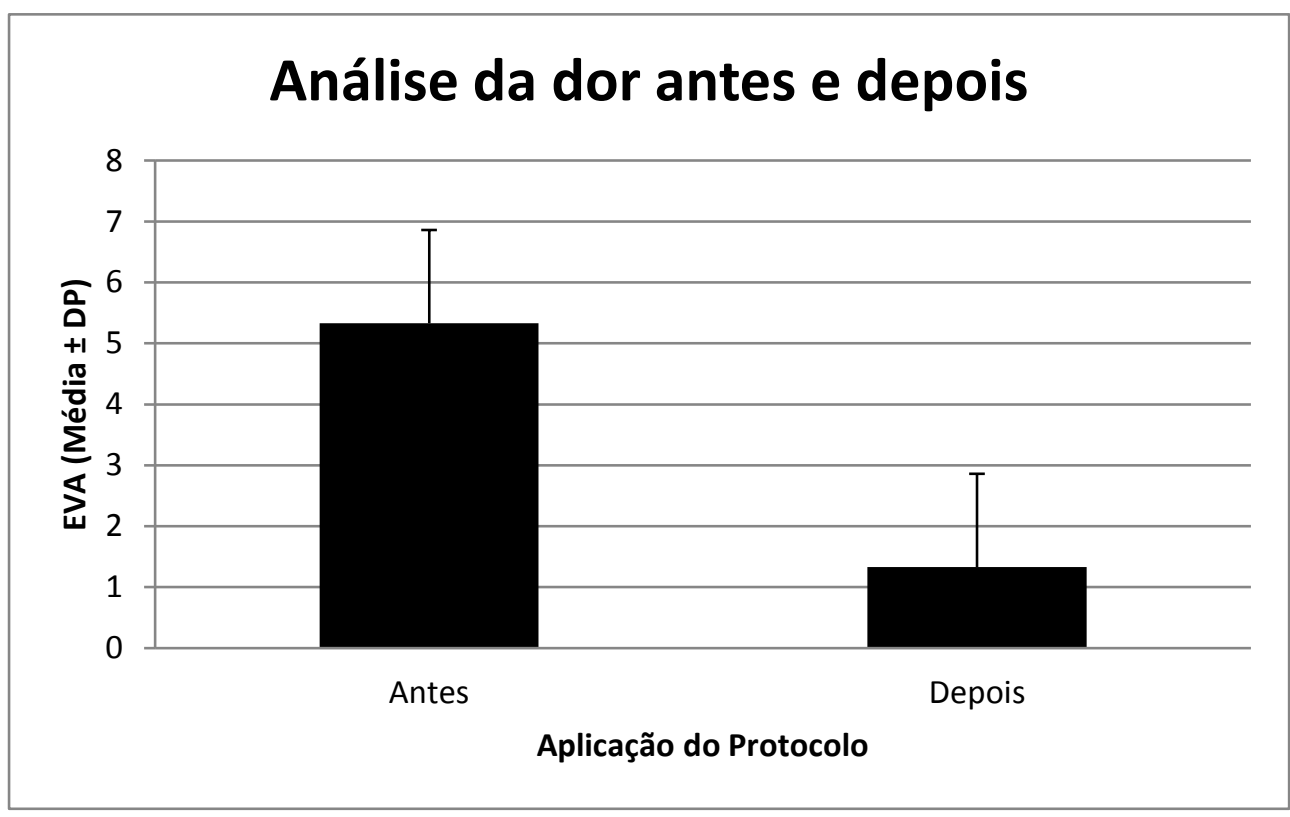




\section{Artigo Original}

\section{Saúde Funcional}

Figura 3. Resultados de força abdominal. Para pacientes do sexo feminino, na faixa etária 30-39 anos, foi considerado, com base no número de repetições de abdominais por minuto: Excelente: > 29; Acima da média: 24-28; Média 20-23; Abaixo da média: 15-19; Fraco <14. Já para o paciente do sexo masculino na faixa etária de 20-29 anos, foi considerado: Excelente: > 43; Acima da média: 37-42; Média 33-36; Abaixo da média: 29-32; Fraco <28.

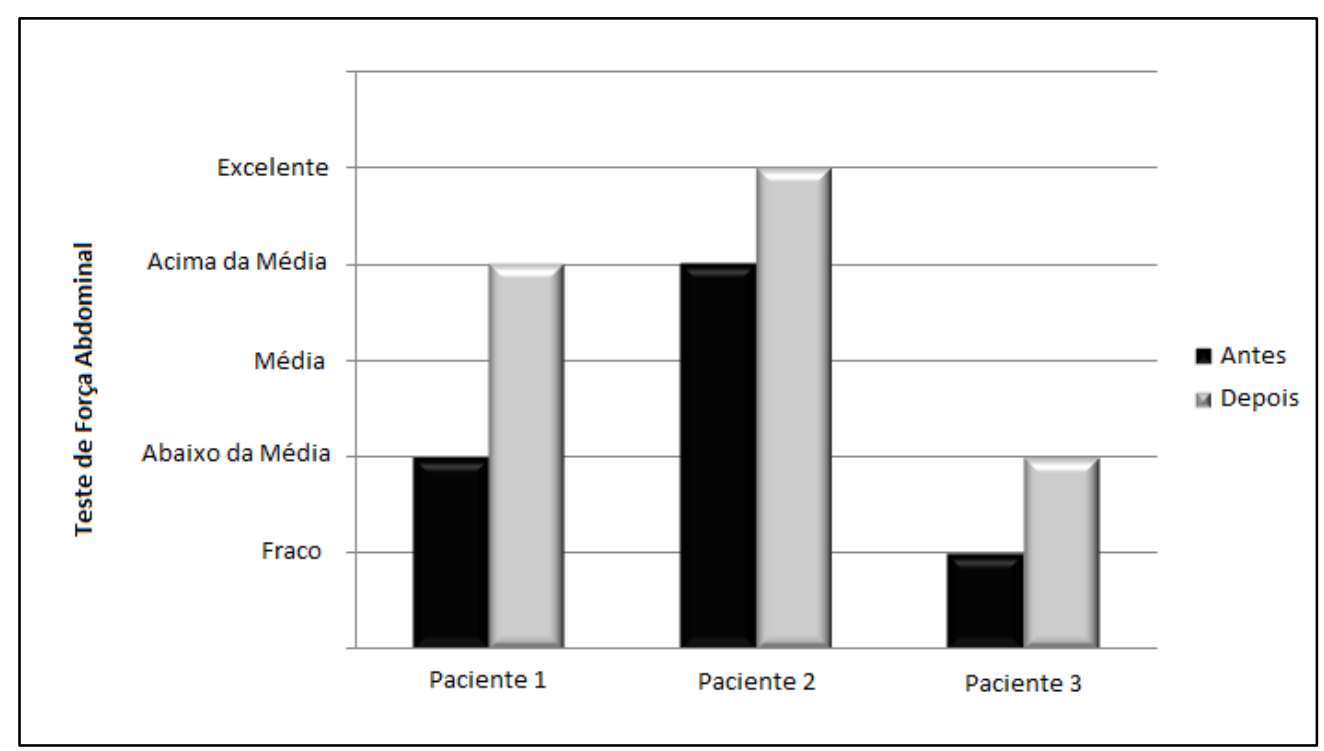

\section{DISCUSSÃO}

No presente estudo podemos observar que após a realização do Método Pilates houve uma melhora da dor lombar, avaliado através da EVA. Em relação aos resultados referentes à aplicação do questionário nórdico de sintomas osteomusculares, foi verificado que, com o ganho de força muscular através do Método Pilates, pode haver uma redução dos sintomas dolorosos da região da coluna lombar. Em consequência, o fortalecimento da musculatura pode levar ao alívio da irradiação dolorosa para membros inferiores. Em outro estudo realizado por Kolinyak e colaboradores ${ }^{19}$ foi demonstrado que com a prática do Método Pilates, houve melhora da dor lombar pelo ganho de força em 20 pacientes. O método pode ser um eficiente recurso terapêutico para fortalecer a musculatura extensora do tronco, favorecendo, dessa forma, uma melhora dos distúrbios da coluna lombar, com o ganho de equilíbrio entre os músculos que realizam extensão e flexão do tronco. 
De acordo com os nossos resultados, Conceição e Mergener ${ }^{26}$ também demonstraram uma melhora na dor lombar, através da EVA, após a aplicação do Método Pilates em sete pacientes. Uma possível explicação para a melhora da dor dos pacientes é que o Pilates é um Método que trabalha com exercícios musculares de baixo impacto contracional, fortalecendo intensamente a musculatura abdominal, proporcionando maior estabilidade para o tronco, prevenindo e tratando quadros álgicos lombares ${ }^{27}$. Juntos estes resultados são importantes para o desenvolvimento de um plano de intervenção e tratamento para os pacientes com dor lombar. Além disso esses dados sugerem que o Método Pilates pode ser uma boa opção para tratamento da dor lombar.

Os resultados do presente estudo demonstraram que os três pacientes avaliados apresentaram ganho de força abdominal após a aplicação do Método Pilates. Ferreira e colegas ${ }^{28}$ demonstraram que o treinamento de força com ênfase sobre a musculatura abdominal foi eficiente em promover aumento da resistência muscular localizada em 12 pacientes do sexo feminino, com idade entre 25 e 40 anos.

O sistema básico do Método Pilates inclui um programa de exercícios que fortalecem a musculatura abdominal e paravertebral, bem como os de flexibilidade da coluna, além de exercícios para o corpo como um todo. Kloubec ${ }^{29}$, ao aplicar o treinamento do Método Pilates em 50 indivíduos, realizado duas vezes por semana, observou que o período de 12 semanas foi suficiente para promover aumento significativo da resistência abdominal nos participantes. Para $\mathrm{Craig}^{30}$, qualquer debilidade nos músculos abdominais afeta a estabilidade da coluna lombar, podendo causar dor. Quando os músculos abdominais são fortes, eles mantêm a coluna adequadamente alinhada, suportam e distribuem o estresse localizado nela, sendo o músculo transverso do abdome o principal responsável pela estabilização da coluna lombar, desempenhando um importante fator para uma boa postura.

\section{CONCLUSÃO}

Com base nos resultados obtidos neste estudo, conclui-se que a utilização do Método Pilates foi eficiente em promover a melhora da dor lombar e força muscular abdominal nos participantes da pesquisa. Contudo, é importante a realização de novos estudos com amostras maiores para o melhor entendimento dos mecanismos 


\section{Artigo Original}

\section{Saúde Funcional}

do Método Pilates, possibilitando, assim, que a aplicação do Método Pilates seja futuramente utilizada como terapêutica para as discopatias degenerativas lombares.

\section{REFERÊNCIAS}

1. Hadjipavlou AG, Tzermiadianos MN, Bogduk N, Zindrick MR. The pathophysiology of disc degeneration: a critical review. $\mathrm{J}$ bone joint surg br. 2008;90(10):1261-70.

2. Bono CM, Lee CK. Critical analysis of trends in fusion for degenerative disc disease over the past 20 years: influence of technique on fusion rate and clinical outcome. Spine. 2004;29(4):455-63; discussion Z5.

3. Polly DW, Santos ER, Mehbod AA. Surgical treatment for the painful motion segment: matching technology with the indications: posterior lumbar fusion. Spine. 2005;30(16 Suppl):S44-51.

4. Clark CR. Cervical spondylotic myelopathy: history and physical findings. Spine. $1988 ; 13(7): 847-9$.

5. Connell MD, Wiesel SW. Natural history and pathogenesis of cervical disk disease. Orthop clin north am. 1992;23(3):369-80.

6. Law MD, Bernhardt M, White AA. Evaluation and management of cervical spondylotic myelopathy. Instr course lect. 1995;44:99-110.

7. Ono K, Ebara S, Fuji T, Yonenobu K, Fujiwara K, Yamashita K. Myelopathy hand. New clinical signs of cervical cord damage. J bone joint surg br. 1987;69(2):2159.

8. Santos M. Hérnia de Disco: uma revisão clínica, fisiológica e preventiva. Lecturas: educación física y deportes. 2003;9(65):1.

9. Waddell G. The back pain revolution. 2nd ed. London: Churchill Livingstone; 2004.

10. Fardon DF, Milette PC. Nomenclature and classification of lumbar disc pathology. Recommendations of the Combined task Forces of the North American Spine Society, American Society of Spine Radiology, and American Society of Neuroradiology. Spine. 2001;26(5):E93-e113.

11. Huang W, Han Z, Liu J, Yu L, Yu X. Risk Factors for Recurrent Lumbar Disc Herniation: A Systematic Review and Meta-Analysis. Medicine. 2016;95(2):e2378.

12. Morgan-Hough CV, Jones PW, Eisenstein SM. Primary and revision lumbar discectomy. A 16-year review from one centre. J bone joint surg br. 2003;85(6):871-4. 


\section{Artigo Original \\ Saúde Funcional}

13. Wells C, Kolt GS, Bialocerkowski A. Defining Pilates exercise: a systematic review. Complement ther med. 2012;20(4):253-62.

14. Rydeard R, Leger A, Smith D. Pilates-based therapeutic exercise: effect on subjects with nonspecific chronic low back pain and functional disability: a randomized controlled trial. J orthop sports phys ther. 2006;36(7):472-84.

15. Oyarzo CA, Villagran CR, Silvestre RE, Carpintero P, Berral FJ. Postural control and low back pain in elite athletes comparison of static balance in elite athletes with and without low back pain. J back musculoskelet rehabil. 2014;27(2):141-6.

16. Muscolino JE, Cipriani S. Pilates and the "powerhouse": II. J bodyw mov ther. $2004 ; 8(2): 122-30$.

17. Muscolino JE, Cipriani S. Pilates and the "powerhouse": I. J bodyw mov ther. $2004 ; 8(1): 15-24$.

18. Carvalho DA, Lima IAX. Os princípios do método pilates no solo na lombalgia crônica [Monografia]. Tubarão: Universidade do Sul de Santa Catarina; 2006.

19. Kolyniak IEGG, Cavalcanti SMB, Aoki MS. Avaliação isocinética da musculatura envolvida na flexão e extensão do tronco: efeito do método Pilates ${ }^{\circledR}$. Rev bras med esporte. 2004;10(6):487-90.

20. Anderson BD, Spector A. Introduction to Pilates-based rehabilitation. Orthop clin north am. 2000;9(3):395-410.

21. Souma ML, Cabaniss CD, Nataraj A, Khan Z. The Valsalva maneuver: a test of autonomic nervous system function in pregnancy. Am j obstet gynecol. $1983 ; 145(3): 274-8$.

22. Supik LF, Broom MJ. Sciatic tension signs and lumbar disc herniation. Spine. 1994;19(9):1066-9.

23. Barros EN, Alexandre NM. Cross-cultural adaptation of the Nordic musculoskeletal questionnaire. Int nurs rev. 2003;50(2):101-8.

24. Sousa FAEF. Dor: o quinto sinal vital. Rev latino-am enfermagem. 2002;10:4467.

25. Knudson D. Issues in Abdominal Fitness: Testing and Technique. JOPERD. $1999 ; 70(3): 49-55$.

26. Conceição JS, Mergener CR. Eficácia do método Pilates no solo em pacientes com lombalgia crônica: relato de casos. Rev dor. 2012;13(4):385-8.

27. Adams MA. Biomechanics of back pain. Acupunct med. 2004;22(4):178-88. 
Artigo Original

Saúde Funcional

28. Ferrreira CB, Aidar FJ, Novaes GdS, Vianna JM, Carneiro AL, Menezes LdS. O método Pilates $\AA$ sobre a resistência muscular localizada em mulheres adultas. Motricidade. 2007;3(4):76-81.

29. Kloubec JAA. Pilates for improvement of muscle endurance, flexibility, balance, and posture. J strength cond res. 2010;24(3):661-7.

30. Craig C. Pilates com a Bola. 2 ed. ed. São Paulo: Phorte; 2004. 